\title{
Distribuição de Paravelia recens (Drake \& Harris, 1935) (Hemíptera, Heteroptera, Veliidae) em Guzmania brasiliensis Ule, 1907 (Bromeliaceae) na Reserva Florestal Adolpho Ducke, Amazonas, Brasil ${ }^{1}$
}

\author{
Domingos Leonardo Vieira PEREIRA², Ulisses Gaspar NEISS², Ruth Leila Menezes FERREIRA² \\ RESUMO \\ Este é o primeiro registro de Paravelia recens (Drake \& Harris, 1935) (Heteroptera: Veliidae) em fitotelmata de Guzmania brasiliensis \\ Ule, 1907 (Bromeliaceae). O estudo foi conduzido na Reserva Florestal Adolpho Ducke, localizada na rodovia AM $010 \mathrm{~km} \mathrm{26,}$ \\ Manaus, Amazonas. Foram realizadas doze amostragens, entre abril de 2003 e abril de 2005, seis no período chuvoso e seis no \\ período de seca. Para cada amostragem, 12 bromélias foram analisadas, seis terrestres e seis epífitas, totalizando 144 amostras. Com \\ exceção das amostras de março e outubro de 2004, 94 espécimes de P. recens foram encontrados. O número de indivíduos coletados \\ foi semelhante nos estratos amostrados, sendo 46 terrestres e 48 epífitas. No período chuvoso foi encontrado um maior número de \\ P. recens (teste-t entre períodos; $\mathrm{t}=2,57 ; \mathrm{p}=0,011$ ), assim como a abundância de indivíduos esteve positivamente correlacionada com \\ o volume de água por bromélia $\left(\mathrm{r}_{\mathrm{s}}=0,18 ; \mathrm{p}=0,033\right)$. Este fato pode ser explicado pelo maior aporte de água no período chuvoso \\ acarretando o aumento do volume médio de água nas bromélias.
}

PALAVRAS-CHAVE

Amazônia Central; Guzmania brasiliensis; Paravelia recens; fitotelmata.

\section{Distribution of Paravelia recens (Drake \& Harris, 1935) (Hemiptera, Heteroptera, Veliidae) in Guzmania brasiliensis Ule, 1907 (Bromeliaceae) at Adolpho Ducke Forest Reserve, Amazonas, Brazil.}

\section{ABSTRACT}

This is the first record of Paravelia recens (Drake \& Harris, 1935) (Heteroptera: Veliidae) in phytotelmata of Guzmania brasiliensis Ule, 1907 (Bromeliaceae). The study was conduced at Adolpho Ducke Forest Reserve, located on road AM 010 Km 26, Manaus, Amazonas. Twelve samplings were taken between April 2003 and April 2005, namely, six in the wet season and six in the dry season. Twelve bromeliads were analysed for each sampling, six terrestrials and six epiphytics, totaling 144 samples. With the exception of the 2004 March and October samples, 94 specimens of $\mathrm{P}$. recens were found. The number of individuals was similar in the stratums sampled, accounting for 46 terrestrials and 48 epiphytics. P. recens were found in great abundance in the wet season ( $t$-test between season; $t=2,57 ; p=0,011)$, and the abundance of individuals was positively correlated with water volume by bromeliad $\left(r_{s}=0,18 ; p=0,033\right)$. This fact could be explained by the major input of water in the wet season, causing the increase of mean water volume by bromeliad.

\section{KEY-WORDS}

Central Amazonia; Guzmania brasiliensis; Paravelia recens; phytotelmata.

\footnotetext{
1 Projeto de Pesquisa Integrada (PPI 1-0605).

2 Instituto Nacional de Pesquisas da Amazônia, Coordenação de Pesquisas em Entomologia, Divisão de Curso em Entomologia, Av. André Araújo, 2936, Petrópolis, Caixa Postal, 478, 69011-970, Manaus/AM. Fone: (92)3643-3201.e-mail: dleo@inpa.gov.br; ulisses@inpa.gov.br; ruth@inpa.gov.br
} 


\section{INTRODUÇÃO}

Microvelia Westwood, 1834 e Paravelia Breddin, 1898 (Heteroptera: Veliidae) são encontrados em fitotelma na Região Neotropical, sendo que existem quatro espécies de Paravelia restritas a este habitat (Drake \& Hussey, 1954; Polhemus \& Polhemus, 1991). No Brasil, Paravelia é representado por duas espécies: P. manausana Polhemus \& Polhemus, 1984, coletada em Vriesia splitgerberi Ruby Branga (Bromeliaceae), registrada para Manaus, Amazonas, e P. recens (Drake \& Harris, 1935), ocorrendo nos estados do Amazonas em $V$. splitgerberi e no Pará em Aechmea fulgens Brongniart (Bromeliaceae) (Polhemus \& Polhemus, 1991). P. manausana e $P$. recens são espécies muito semelhantes, podendo ser diferenciadas pelos caracteres morfológicos utilizados na chave apresentada por Polhemus \& Polhemus (1991). Os mesmos autores citaram a distribuição de P. recens de Honduras à Bolívia, associada às bromélias terrestres e epífitas de diferentes gêneros e espécies.

Polhemus \& Polhemus (1991) relataram um baixo número de indivíduos por bromélia, geralmente um ou dois e, baixa freqüência de ocorrência, estando ausentes na maioria das bromélias amostradas. Quando presentes tendem à agregação em certas bromélias onde já foram encontrados mais de 12 indivíduos, demonstrando uma distribuição não uniforme.

A distribuição de espécies de invertebrados em fitotelmatas pode ser afetada por fatores bióticos como predação e competição (Srivastava \& Lawton, 1998; Fincke, 1992; Yanoviak, 1999a, 2001a), bem como fatores abióticos como tamanho, volume, pH e temperatura da água, disponibilidade de nutrientes e altura do habitat em relação ao solo (Fincke, 1998, 1999; Yanoviak, 1999b). Culicidae, por exemplo, são presas potenciais de velídeos em fitotelmatas (Frick, 1949; Yanoviak, 1999c; Kitching, 2000). Através de experimentos em laboratório e observações em campo, Yanoviak (1999c) mostrou que Microvelia cavicola Polhemus e $P$. myersi Hungerford, espécies de concavidades em árvores, alimentam-se de mosquitos adultos recém emergidos e de outros insetos capturados na superfície da água. No entanto, não há relatos sobre o hábito alimentar de $P$. recens em bromélias.

Este trabalho registra a ocorrência de $P$. recens em $G$. braziliensis, a abundância de indivíduos entre bromélias epífitas e terrestres e entre as estações seca e chuvosa, além de caracterizar o habitat bromelícola, através de dados abióticos como volume, $\mathrm{pH}$ e temperatura da água.

\section{MATERIAL E MÉTODOS}

O estudo foi realizado na Reserva Florestal Adolpho Ducke, localizada na rodovia AM $010 \mathrm{~km} 26$ (02055'51"S; 5958'59”W), Manaus, Amazonas, em área de floresta de campinarana parcialmente fechada, entre áreas de baixio e vertente, com solo de areia branca e bastante acúmulo de serrapilheira. De acordo com Ribeiro et al. (1999), nesse tipo de ambiente, há poucas árvores de grande porte, o dossel tem entre 15 e 25 m e há alta diversidade de epífitas. Entre abril de 2003 e abril de 2005, foram realizadas doze amostragens: seis no período chuvoso (abril, maio e junho de 2003 e março de 2004, março e abril de 2005) e seis no período de seca (agosto, setembro e outubro de 2003, agosto, setembro e outubro de 2004). Em cada coleta foram analisadas, ao acaso, 12 bromélias Guzmania brasiliensis Ule, 1907 (Figura 1), seis terrestres e seis epífitas, na altura de $3 \mathrm{~m}$, totalizando 144 amostras.

No campo, cada amostra foi rotulada e acondicionada em sacos plásticos para serem transportadas para o laboratório onde foram feitas a triagem e a fixação dos espécimes em álcool 80\%. Para caracterizar o habitat foi medido o volume $(\mathrm{ml}), \mathrm{pH}$ e temperatura da água $\left({ }^{\circ} \mathrm{C}\right)$. Dados de temperatura ambiente e precipitação pluviométrica, fornecidos pela estação experimental da EMPRAPA/AM, localizada na rodovia AM 010, km 30, foram utilizados para caracterizar cada período sazonal.

A Correlação de Spermann e o Teste-t (Student) foram utilizados para verificar a relação da abundância de espécimes com o $\mathrm{pH}$, volume e temperatura da água e período anual. Para todos os testes estatísticos foi utilizado $\mathrm{p}=0,05$.

O material entomológico foi depositado na Coleção de Invertebrados do INPA e exsicatas de G. brasiliensis no Herbário do INPA.

\section{RESULTADOS E DISCUSSÃO}

Foram analisadas 144 bromélias. Nove (6,25\%) foram encontradas sem água e $50(34,72 \%)$ continham representantes de $P$. recens, totalizando 94 indivíduos, com uma média de 0,65 $( \pm 1,12)$ indivíduos por bromélia. Não houve coexistência de $P$. manausana e $P$. recens em $G$. brasiliensis e não foi encontrado mais do que seis indivíduos por bromélia.

Pela primeira vez é registrada a ocorrência de $P$. recens em $G$.

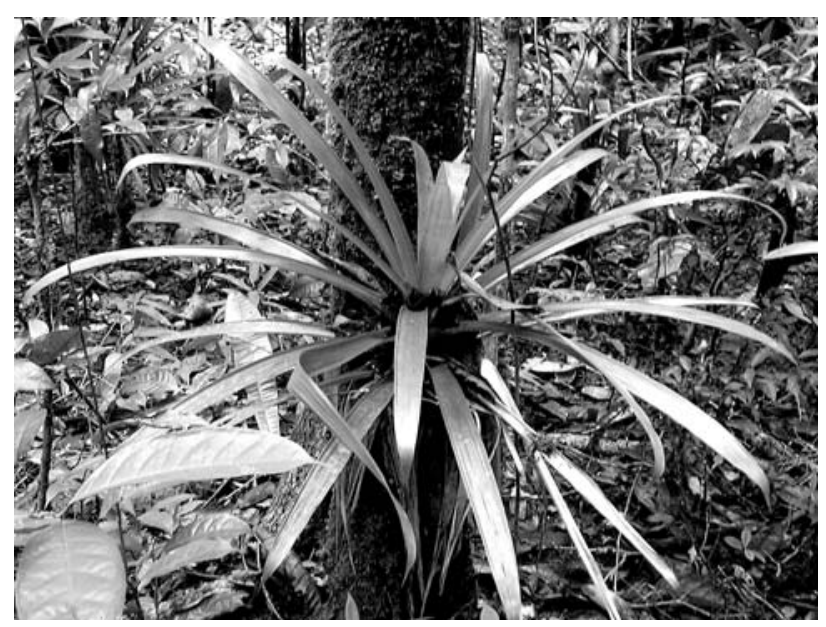

Figura 1 - Vista geral de epífita de Guzmania brasiliensis (Bromeliaceae) da Reserva Florestal Adolph Ducke. 
brasiliensis, tanto em estrato arbóreo como terrestre, além de estar presente durante todos os meses amostrados com exceção de março e outubro de 2004. A Tabela 1 mostra dados de média e desvio padrão do número de indivíduos, volume, $\mathrm{pH}$ e temperatura da água acumulada na bromélia, além de dados médios de temperatura ambiente e precipitação pluviométrica total de cada período amostrado.

Não houve diferença significativa no número de indivíduos entre bromélias epífitas e terrestres (epífitas: $\mathrm{n}=48, \bar{x}=0,66$ $\pm 1,24$; terrestres: $\mathrm{n}=46, \bar{x}=0,64 \pm 1$; teste- $\mathrm{t}$; $\mathrm{t}=-0,332 ; \mathrm{p}=$ $0,74)$. Do mesmo modo, a diferença entre os volumes de água das bromélias epífitas e terrestres não foi significativa (teste- $\mathrm{t} ; \mathrm{t}=$ $-0,878 ; p=0,38)$, sugerindo que a diferença de três metros entre bromélias epífitas e terrestres não é suficiente para diferenciar os estratos. Yanoviak (1999c) em um estudo com M. cavicola em fitotelmata de ocos de árvores, no Panamá, também relatou a baixa freqüência deste velídeo em criadouros naturais. Além disso, o mesmo autor demonstrou que $M$. cavicola possui uma tendência a diminuir a abundância em ocos de árvores com o aumento da altura.
A soma total de indivíduos e do volume de água coletado nas bromélias no período chuvoso foi 64 (68\%) e $8.975 \mathrm{ml}$ (57\%), respectivamente; enquanto que no período seco foi de $30(32$ $\%$ e $6.738 \mathrm{ml}(43 \%)$. A diferença entre o número de indivíduos por período foi significativa (teste-t; $t=2,57 ; \mathrm{p}=0,011$ ), assim como a abundância de indivíduos esteve positivamente correlacionada com o volume de água por bromélia $\left(\mathrm{r}_{\mathrm{s}}=0,18 ; \mathrm{p}\right.$ = 0,033). Do mesmo modo, Yanoviak (1999c) também verificou que a abundância de $M$. cavicola em buracos naturais em árvores aumentou com o volume. Este fato pode ser explicado pelo maior aporte de água no período chuvoso acarretando o aumento do volume médio de água nas bromélias.

Esse aumento do volume pode proporcionar uma expansão do habitat disponível e de nutrientes provenientes da lixiviação, favorecendo uma maior abundância de invertebrados associados a esses fitotelmatas (Frank, 1983; Armbruster et al., 2002; Ospina-Bautista et al. 2004). Mosquitos, por exemplo, são comuns em fitotelmatas e tendem a aumentar a abundância com o aumento do volume de água (Lounibos, 1983; Frank, 1983;

Tabela 1 - Média e desvio padrão do número de $P$. recens, volume $(\mathrm{mL})$, pH e temperatura d'água $\left({ }^{\circ} \mathrm{C}\right)$ por bromélia epífita $(\mathrm{E}) /$ terrestre $(\mathrm{T})$ para cada mês de amostragem (12 bromélias por amostra, seis epífitas e seis terrestres, totalizando 144 bromélias). Dados da média de temperatura ambiente e precipitação pluviométrica total também estão relacionados para cada período de coleta (entre Abr/03 e Abr/05) na Reserva Adolpho Ducke, Manaus, AM.

\begin{tabular}{|c|c|c|c|c|c|c|c|}
\hline \multirow{2}{*}{ Mês } & \multirow{2}{*}{$\begin{array}{l}\text { Temperatura ambiente } \\
\left({ }^{\circ} \mathrm{C}\right)\end{array}$} & \multirow{2}{*}{$\begin{array}{l}\text { Precipitação total } \\
(\mathrm{mm})\end{array}$} & \multirow{2}{*}{ Estratos } & \multirow{2}{*}{ n (P. recens) } & \multicolumn{3}{|c|}{ G. brasiliensis } \\
\hline & & & & & Volume & Temperatura $\left({ }^{\circ} \mathrm{C}\right)$ & $\mathrm{pH}$ \\
\hline \multirow{2}{*}{ Abril/03 } & \multirow{2}{*}{$27,2 \pm 1,2$} & \multirow{2}{*}{386,3} & $E$ & $2,3 \pm 1,21$ & $195 \pm 55,8$ & $27 \pm 0,6$ & $6 \pm 0,6$ \\
\hline & & & $\mathrm{T}$ & $1 \pm 1,26$ & $228,3 \pm 112,8$ & $27,2 \pm 0,4$ & $5,5 \pm 0,5$ \\
\hline \multirow{2}{*}{ Maio/03 } & \multirow{2}{*}{$27,1 \pm 1,2$} & \multirow{2}{*}{323} & $E$ & $0,7 \pm 0,8$ & $108,3 \pm 37,6$ & $26,8 \pm 0,4$ & $5,1 \pm 0,3$ \\
\hline & & & T & $0,6 \pm 0,8$ & $230 \pm 73,6$ & $27,4 \pm 0,5$ & $5,6 \pm 0,3$ \\
\hline \multirow{2}{*}{ Junho/03 } & \multirow{2}{*}{$27,4 \pm 0,8$} & \multirow{2}{*}{168,8} & $E$ & $2,7 \pm 2,3$ & $175 \pm 236,1$ & $27 \pm 0$ & $4,9 \pm 0,2$ \\
\hline & & & T & $2,7 \pm 1$ & $108,3 \pm 58,5$ & $27,2 \pm 0,4$ & $5,2 \pm 0,2$ \\
\hline \multirow{2}{*}{ Agosto/03 } & \multirow{2}{*}{$28 \pm 0,8$} & \multirow{2}{*}{121,2} & $E$ & $0,2 \pm 0,4$ & $87,5 \pm 37,9$ & $27,8 \pm 0,4$ & $4,8 \pm 0,4$ \\
\hline & & & $T$ & $0,8 \pm 0,7$ & $74,2 \pm 30,1$ & $28 \pm 0,8$ & $4,7 \pm 0,3$ \\
\hline \multirow{2}{*}{ Setembro/03 } & \multirow{2}{*}{$28,4 \pm 0,9$} & \multirow{2}{*}{159,7} & $E$ & $0,3 \pm 0,8$ & $100 \pm 35,8$ & $25 \pm 0$ & $4,7 \pm 0,4$ \\
\hline & & & T & $0,3 \pm 0,5$ & $74 \pm 25,1$ & $26,4 \pm 1,9$ & $4,4 \pm 0,6$ \\
\hline \multirow{2}{*}{ Outubro/03 } & \multirow{2}{*}{$28,6 \pm 1,2$} & \multirow{2}{*}{140,1} & $E$ & $0,8 \pm 1,2$ & $230 \pm 131,6$ & $27,6 \pm 0,8$ & $5,2 \pm 0,9$ \\
\hline & & & $\mathrm{T}$ & $0,8 \pm 1,2$ & $99,2 \pm 65,3$ & $28,2 \pm 0,4$ & $4,7 \pm 0,7$ \\
\hline \multirow{2}{*}{ Março/04 } & \multirow{2}{*}{$26,7 \pm 0,9$} & \multirow{2}{*}{462,8} & $E$ & 0 & $114 \pm 58,6$ & $28,6 \pm 0,5$ & $5,2 \pm 0,8$ \\
\hline & & & T & 0 & $131 \pm 81$ & $27,8 \pm 0,4$ & $5,1 \pm 0,6$ \\
\hline \multirow{2}{*}{ Março/05 } & \multirow{2}{*}{$27 \pm 0,9$} & \multirow{2}{*}{437,6} & $E$ & $0,3 \pm 0,8$ & $76 \pm 46,2$ & $27,8 \pm 0,4$ & $4,5 \pm 0,4$ \\
\hline & & & $T$ & 0 & $66,7 \pm 37,6$ & $28 \pm 0,8$ & $4,5 \pm 0,5$ \\
\hline \multirow{2}{*}{ Abril/05 } & \multirow{2}{*}{$27,6 \pm 0,6$} & 474 & $E$ & $0,2 \pm 0,4$ & $56,3 \pm 47,3$ & $27,7 \pm 0,9$ & $4,5 \pm 0,5$ \\
\hline & & $4 / 4$ & T & $0,3 \pm 0,8$ & $67,5 \pm 24,2$ & $27,5 \pm 0,5$ & $4,6 \pm 0,4$ \\
\hline Agnsto/04 & $278+11$ & 1589 & $E$ & $0,5 \pm 0,8$ & $106 \pm 56,4$ & $28 \pm 0,7$ & $4,6 \pm 0,5$ \\
\hline Agusiovit & $2 i, 0 \pm 1,1$ & 100,5 & T & $0,5 \pm 0,8$ & $95 \pm 72,8$ & $27,8 \pm 0,7$ & $4,6 \pm 0,5$ \\
\hline Outuhro/04 & $284+13$ & 1785 & $E$ & 0 & $102,5 \pm 38,2$ & $27,8 \pm 0,4$ & $4,4 \pm 0,4$ \\
\hline 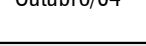 & $20,7-1,0$ & 110,0 & $\mathrm{~T}$ & 0 & $60,8 \pm 7,4$ & $28,3 \pm 0,8$ & $4 \pm 0,1$ \\
\hline Novemhro/04 & $288+1$ & 1053 & $E$ & 0 & $104,7 \pm 65,3$ & $28 \pm 0,6$ & $4,4 \pm 0,4$ \\
\hline 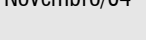 & $20,0-1$ & 100,0 & $T$ & $0,7 \pm 0,5$ & $69,2 \pm 52,5$ & $28,5 \pm 0,5$ & $4,4 \pm 0,4$ \\
\hline
\end{tabular}

$T=$ terrestre; $E=$ epifita 
Fish, 1983), desse modo o aumento do volume de água facilitaria a coexistência de um maior número de Heteroptera nesses ambientes.

A abundância de indivíduos por bromélia esteve positivamente correlacionada ao $\mathrm{pH}\left(\mathrm{r}_{\mathrm{s}}=0,29 ; \mathrm{p}=0,005\right)$ e inversamente correlacionada à temperatura da água $\left(\mathrm{r}_{\mathrm{s}}=-0,26 ; \mathrm{p}\right.$ $=0,02)$. Esses valores físico-químicos da água concordam com outros estudos de macroinvertebrados em fitotelmata de bromélia (Laessle, 1961), em ocos de árvores e recipientes artificiais (Yanoviak, 1999b, 2001b) e, também na Bromeliaceae Tillandsia turneri Baker (Ospina-Bautista et al. 2004). As variáveis provavelmente não estão influenciando diretamente os velídeos, já que esses ocupam o "pleuston", mas indiretamente, podem afetar a disponibilidade de presas, influenciando a ocupação e abundância dos mesmos.

\section{AGRADECIMENTOS}

À bolsista Sharlene Roberta da Silva Torreias pelo material coletado.

\section{BIBLIOGRAFIA CITADA}

Armbruster, P.; Hutchinson, R.A.; Cotgreave, P. 2002. Factors influencing community structure in a South American tank bromeliad fauna. Oikos, 96: 225-234.

Drake, C.J.; Hussey, R.F. 1954. Notes on some American Veliidae (Hemiptera) with the description of two new Microvelias from Jamaica. Florida Entomologist, 27: 133-138.

Fincke, O.M. 1992. Consequences of larval ecology for territoriality and reproductive success of a neotropical damselfly. Ecology, 73: 449-462.

Fincke, O.M. 1998. The population ecology of Megaloprepus coerulatus and its effect on species assemblages in water-filled tree holes. In: Dempster, J.P.; Mclean, I.F.G. (Eds). Insect populations in theory and in practice. Kluwer Academic, Dordrecht. p. 391-416.

Fincke, O.M. 1999. Organization of predator assemblages in Neotropical tree holes: effects of abiotic factors and priority. Ecological Entomology, 24: 13-23.

Fish, D. 1983. Phytotelmata: Flora and Fauna, In: Frank, J.H.; Lounibos, L.P. (Eds.). Phytotelmata: Terrestrial Plants as Host for Aquatic Insect Communities. Medford, New Jersey, Plexus. p. 128.

Frank, J.H. 1983. Bromeliad phytotelmata and their biota, especially mosquitoes, In: Frank, J.H.; Lounibos, L.P. (Eds.). Phytotelmata: Terrestrial Plants as Host for Aquatic Insect Communities. Medford, New Jersey; Plexus. p.101-128.

Frick, K.E. 1949. The biology of Microvelia capitata Guerin, 1857, in the Panama Canal Zone andits role as a predator on anopheline larvae (Veliidae: Hemiptera). Annals of the Entomological Society of America, 42: 77-100.

Kitching, R.L. 2000. Food webs and container habitats: the natural history and ecology of phytotelmata. Cambridge University Press, Cambridge, Inglaterra. 431pp.

Laessle, A.M. 1961. A micro-limnological study of Jamaican bromeliads. Ecology, 42: 499-517.

Lounibos, L.P. 1983. The mosquito community of treeholes in subtropical Florida. In: Frank, J.H.; Lounibos, L.P. (Eds.). Phytotelmata: Terrestrial Plants as Host for Aquatic Insect Communities. Plexus, Medford, New Jersey. p. 223-246.

Ospina-Bautista, F.; Estévez-Varón, J.V.; Betancur, J.; RealpeRebolledo, E. 2004. Estructura y composición de la comunidad de macoinvertebrados acuáticos asociados a Tillandsia turneri Baker (Bromeliaceae) en un bosque alto andino colombiano. Acta Zoológica Mexicana, 20(1): 153-166.

Polhemus, J.T.; Polhemus, D.A. 1991. A review of the veliid fauna of bromeliads, with a key and description of a new species (Heteroptera: Veliidae). Journal of New York Entomological Society, 99(2): 204-216.

Ribeiro, J.E.L. da S.; Hopkins, M.J.G.; Vicentini, A.; Sothers, C.A.; Costa, M.A. da S.; Brito, J.M. de.; Sousa, M.A. de.; Martins, L.H.P.; Lohmann, L.G.; Assunção, P.A.C.L.; Pereira, E. da C.; Silva. C.F. da.; Mesquita, M.R.; Procópio, L.C. 1999. Flora da Reserva Ducke: Guia de identificação das plantas vasculares de uma floresta de terra-firme na Amazônia Central. Instituto Nacional de Pesquisas da Amazônia, Manaus, Amazônas. 799 pp.

Srivastava, D.S.; Lawton, J.H. 1998. Why more productive sites have more species: a experimental test of theory using tree-hole communities. American Naturalist, 116: 770-787.

Yanoviak, S.P. 1999a. Effects of leaf litter species on macroinvertebrate community properties and mosquito yield in Neotropical tree hole microcosms. Oecologia, 120: 147-155.

Yanoviak, S.P. 1999b. Comunity structure in water-filled tree holes of Panama: effects of hole height and size. Selbyana, 20: 106-115

Yanoviak, S.P. 1999c. Distribution and abundance of Microvelia cavicola Polhemus (Heteroptera: Veliidae) on Barro Colorado Island, Panama. Journal of New York Entomological Society, 107: $38-45$.

Yanoviak, S.P. 2001a. Predation, resource availability, and community structure in Neotropical water-filled tree holes. Oecologia, 126: 125-133.

Yanoviak, S.P. 2001b. Container color and location affect macroinvertebrate community structure in artificial treeholes in Panamá. Florida Entomologist, 84(2): 265-271.

Recebido em 15/12/2006

Aceito em 21/12/2006 